\title{
An Energy Consumption Model of Mobile Terminal Software Based on BP Neural Network
}

\author{
L.W. Liu, T.F. Zhan, Z.Y. Cai \\ College of Computer and Information technology \\ China Three Gorges University \\ Yichang, China
}

\begin{abstract}
The energy consumption of software plays an important role in mobile application. Firstly, on the point of measurement, the characteristics of mobile terminal software are studied, and the functional relation between software energy and software characteristic is modeled as a nonlinear function. Secondly, based on BP neural network, an energy consumption model of mobile software is built. After the analysis of software characteristics in system architecture, the training and study mechanism of BP neural network in energy consumption measures is illustrated here. Lastly, BP training to fit the functional relation between software architecture and energy consumption is verified by experimental results.
\end{abstract}

Keywords-mobile commerce; software energy; BP neural network; mobile terminal

\section{INTRODUCTION}

Along with the rapid advance of society and the development of economy, energy problem has become more and more serious, and the energy crisis has become the focus of social attention [1]. Now, the idea of energy saving has deeply rooted in the hearts of people. In energy saving, work and life are most closely related to our energy consumption with the increasing popularity of mobile terminals and all kinds of applications [2,3].

Reference [4] put forward a model of the mobile payment through the research in mobile agents and the existed mobile system. In mobile terminals, software architecture and mobile application are often designed in lightweight [5]. The rapid development of e-commerce is very popular in the world, and reference [6] considers the mobile terminal as an integral role in this respect. Then literature [7] presented a mobile terminal structure layered of a context-aware agent, and reference [8] proposed a model of mobile commerce value net. However, in these researches on mobile terminals and e-commerce, the impact of the software architecture itself on energy consumption is ignored.

This article puts forward a modeling method of the energy consumption of mobile terminal software. On the aspect of mobile architecture, a three level software architecture modelthe component number, average complexity of component interface, average path complexity characteristics, is put forward. Assuming that there is a nonlinear function relationship between these characteristics and software energy consumption, the energy consumption of the mobile terminal software architecture model is established.

\section{ENERGY CONSUMPTION MODELING OF MOBILE TERMINAL}

Figure 1 shows a general system architecture of the mobile terminal software where the lower layers play an important role in the mobile terminal system. Among them, Graphical User Interface (GUI) provides an interface for users and runs on the Operating System Abstraction Layer (OSAL). The Protocol Stack Abstraction Layer (PSAL) refers to the sum of each layer protocol in network reflecting a network file transfer process. Device Abstraction Layer (DAL) is located at the interface between the operating system kernel and hardware circuit layer.

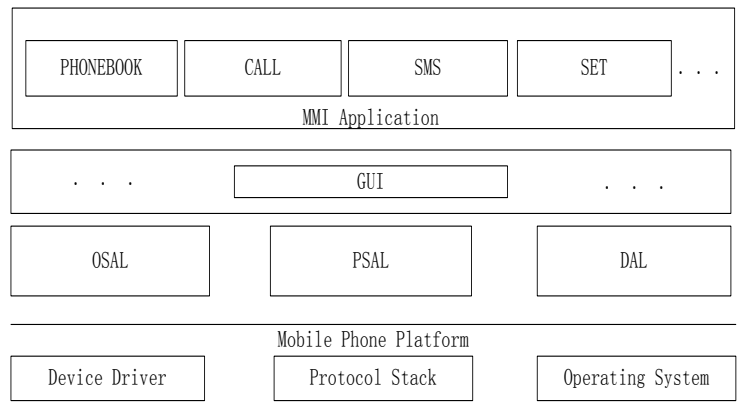

FIGURE I. SOFTWARE ARCHITECTURE MODEL.

Because of the layer model and the software architecture level characteristics, the relationship between the energy consumption and software is considered as a nonlinear function relation. Assuming $E$ is the software energy consumption, $a_{1}$ is the initialization consumption of the software energy, $b_{n}$ is the software characteristic value which is associated with energy consumption of software, and the value of $a_{n}$ represents the parameter of the characteristics of various software, then the E can be expressed as

$$
E=a_{1}+a_{2} b_{1}+a_{3} b_{2}+\ldots+a_{n+1} b_{n}
$$

A lot of mobile operating systems are used such as Androis, ios, Java, windows Mobile, symbian OS etc. There are a lot of mobile software that are related to these Operating systems; people have to download mobile software to their mobile terminals via WIFI from the Internet where there is a variety of Mobile software for people to use. Traditional architecture and energy consumption modeling use linear 
regression method, which mainly consider the characteristics of software on architecture level, the $n_{\text {th }}$ linear software function between energy consumption and the energy consumption model. Through the analysis of some software architecture level characteristics and relationship of energy consumption between different software, we can get a architecture model of energy consumption.

$$
E_{s}=A \times T=f(B) \times T=f\left(L, C, R_{c}, R_{w}, D C\right) \times T
$$

In the formula above, $E_{s}$ is the consumption of the software and $A$ is the average power consumption. $T$ is the software running time and $B$ is some software metrics associated with software power consumption characteristics, and $f$ is the nonlinear function between software power consumption and software related measurement features. This article will use the BP neural network to carry on the fitting. $L$ is the line number of effectively software code, $C$ is component quantity, $R_{c}$ is average complexity of component interface, the $R_{w}$ is average path complexity, and $D C$ is the average component coupling.

\section{The Nonlinear Structure of BP NeURAL NETWORK}

Using BP neural network to realize nonlinear fitting of the specific process is as follows.

For large sample program of 4 level software architecture as shown in Figure 1, the characteristics including effective lines of code, member number, the average complexity of component interface, the average path complexity, and the average component coupling can be measured.

As GUI, OSAL, PSAL,DAL, etc., the 4 software metric values of the characteristics of preprocessing, after processing, can be taken as the input value of the BP neural network for study and update of neutral network. After running a sample program on power simulation experiment platform, the energy consumption values $\mathrm{E}$ of sample process can be calculated, where $E$ will serve as the output value of the BP neural network.

Determining the specific structure of the BP neural network, and it includes the number of hidden layer, hidden layer node number and transfer function of hidden layer and output layer transfer function, etc.

Nowadays, many mobile terminals come with a lot of apps pre-installed, including weather apps, mail apps and games. By inputting sample application of software characteristics value and measured energy consumption values, the BP neural network can be trained and optimized for further determining the BP neural network weights and threshold of hidden layer by training.

The network in BP system refers to the inter-connections between different neurons in all layers in mobile system. The first layer with input neurons can send data via synapses to those in second layer, and then to the last layer via of output neurons more synapses. By feeding into the characteristics of target program to the trained BP neural network together with the predictive value of energy consumption, the actual energy consumption of target program can be compared to verify the validity of the modeling method.

The most important thing in neural networks is its possibility of learning. After determining the various parameters, the network can be tested. Through a large number of sample data input, if the minimum mean square error precision is lower than a pre-defined error, we will get the weights of BP neural network $w_{1}, w_{2}$ and threshold $b_{1}$, $b_{2}$. Lastly, the identification functions of each layer should be defined.

The task of training a neural network model is usually to select one model from a set of remains. Generally speaking, as the single hidden layer in BP neural network, the transfer function of input layer and hidden layer is tansig function, the transfer function of the output layer is purelin function. As Trainlm function, its convergence speed and the approximation error can achieve satisfactory results when the number of hidden layer nodes is 10 , which can be found through many experiments. Therefore, the training function of BP neural network is defined as Trainglm, and the hidden layer node number of training function is 10 .

\section{ENERGY OPTIMIZATION ALGORITHM}

According to characteristics of GUI, OSAL, PSAL,DAL, etc., energy consumption modeling of software architecture level can be divided into the following 4 steps.

Step 1, Initialization.

Based on Figure 1, we assume there is a nonlinear function relationship between energy consumption and software characteristics of architecture level.

Five aspects of system structure and the energy consumption of software are isolated from each other and separated from software characteristics namely effective lines of code, number of components, the average complexity of component interface, the average path complexity, and the average component coupling.

\section{Step 2, Measurement}

By choosing reasonable measurement methods, line number of code, components, the average complexity of component interface, the average path complexity, average measurement of the characteristic components can be coupled in architecture level.

As the number of hidden layer, it is important to determine how much is in hidden layer to determine the network error. However, too many hidden layers will result in complicate network, and the network training time and the fitting time will increase. A three-layer BP network can be completed from any $n$ to $m$ dimensional mapping. Therefore, the number of hidden layer is determined as 1.

Step 3, iteration 
Through the simulation experiment platform of power consumption and a set of benchmark programs, the value of $T S$ and ES can be measured according to the formula (1). The average power consumption during the software running value can be concluded.

$$
P=E_{s} / T
$$

After getting effective architecture characteristic measurements, it is necessary to further determine the structure of the BP neural network. Then it is to determine the number of hidden layer nodes. In the BP neural network, the number of hidden layer nodes has a huge impact on BP network performance.

\section{Step 4, Training and Terminate}

By training the BP neural network to fit the nonlinear correlation functions $f$ of the input values for each software architecture level, characteristics measurement and average power output of the software can be gotten. It is typical to determine the number of nodes in hidden layer by the following empirical formula $n$ :

$$
n=\sqrt{a+b}+i
$$

where $a$ is input layer node number, $b$ is the output layer node number, and $i$ is the constant between 1 and 10 .

\section{EXPERIMENT AND ANALYSIS}

In order to verify the effectiveness of the energy consumption model based on software characteristics, this paper adopts five target programs to verify this model; the program contains a common flow file and more complex MP3 file decoding program. In Win 7, 32bit and Matlab 7.0 environment, through specific environment simulation experiment, assuming the energy level is in the area $[0,1]$, the verification results are shown in Figure 2.

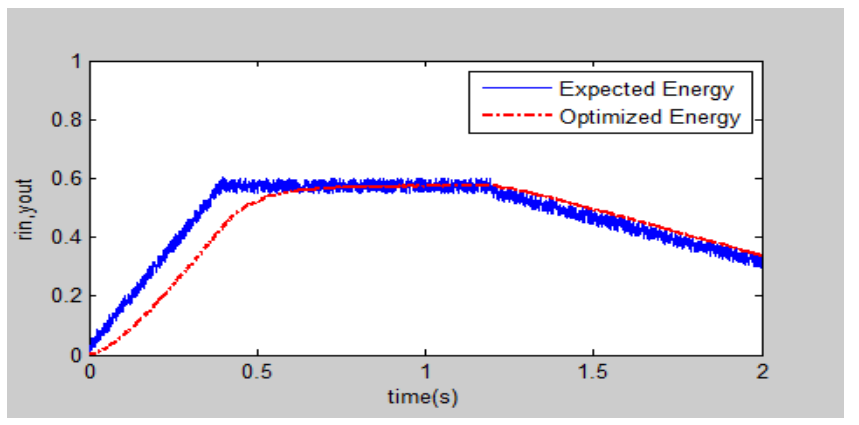

FIGURE II. EXPERIMENT RESULTS.

As we can see from Figure 2, target application data model of expected energy consumption value and the actual energy consumption value of maximum error is $33.4 \%$, the minimum error is $3.1 \%$, and the average error is $17.5 \%$. The optimized energy consumption takes apparent advantages over that of expected energy, especially in step up period of software. It is not hard to see that there is a certain correlation between each characteristic and energy consumption values, and the most obvious characteristic is $L$ which is on behalf of the effective lines of code.

\section{CONCLUSIONS}

This paper establishes a model of energy consumption and applies BP neural network as a function fitting method into energy management. The nonlinear function relationship between software characteristic quantities and software energy consumption is verified by an experiment. The future research is to extend these software characteristic quantities on more complex mobile terminal platform and software environment, and further research on optimization of energy consumption, etc.

\section{ACKNOWLEDGEMENTS}

This research was supported by the National Natural Science Foundation of China (No. 71471102), and Key Projects of Science and Technology Research Program of Hubei Provincial Department of Education in China (Grant No. D20101203).

\section{REFERENCES}

[1] Zhu, Yuhao; Reddi, Vijay Janapa . High-Performance and EnergyEfficient Mobile Web Browsing on Big/Little Systems[C]. 19th IEEE International Symposium on High Performance Computer Architecture (HPCA). (Shenzhen, PEOPLES R CHINA . FEB 23-27, 2013), 2013: 13-24.

[2] Song, Jaeki; Kim, Junghwan; Jones, Donald R.; Application discoverability and user satisfaction in mobile application stores: An environmental psychology perspective[J]. DECISION SUPPORT SYSTEMS, 2014, 59:37-51.

[3] Li, Gangmin; Shen, Zhun. A Middleware Architecture for Price Comparison Service on Mobile Phones [C]. st International Conference on Information Technology and Quantitative Management (ITQM).( Suzhou, PEOPLES R CHINA, MAY 16-18, 2013), 2013,17: 545-553.

[4] Li, Yan; Hu, Xiaoqiang; Zeng, Liang. The Application of Mobile Agent in Mobile Payment[C]. International Conference on Computer Science and Network Technology (ICCSNT).( Harbin Normal Univ, Harbin, PEOPLES R CHINA, DEC 24-26, 2011), 2012,1-4:1612-1616.

[5] Ruiz-Martinez, A.; Inmaculada Marin-Lopez, C; Sanchez-Martinez,D; SIPmsign:a lightweight mobile signature service based on the Session Initiation Protocol[J].SOFTWARE-PRACTICE \& EXPERIENCE,2014,44(5):511-535.

[6] Wang, Jiangjing; Jiang, Jiulei; He, Feng. Problem and Countermeasure on the Development of Mobile Electronic Commerce in China[C]. International Conference on Instrumentation, Measurement, Circuits and Systems (ICIMCS 2011).( Hong Kong, PEOPLES R CHINA, DEC 1213, 2011),2012,127:861-866.

[7] Peng Min-jing; Li Bo; Liu Meng. A Layered Context-aware Agent for Mobile Applications based on Users Needs Hierarchy [C]. International Conference on Mechatronics and Semiconductor Materials (ICMSCM 2013).( Xian, PEOPLES R CHINA, SEP 28-29, 2013), 2014,846-847: 1689-1692 .

[8] Sun, Jie; Ren, Wei'anResearch on Mobile Business Value Network and Model Construction [C]. International Conference on Mechatronics and Information Technology (ICMIT 2013).( Guilin, PEOPLES R CHINA, OCT 19-20, 2013 ), 2014,462-463: 849-855. 